\title{
Differential Invariants for Parametrized Projective Surfaces
}

\author{
Gloria Marí Beffa and Peter J. Olver ${ }^{1}$
}

\begin{abstract}
We classify the differential invariants and moving frames for surfaces in projective space under the action of the projective group. The role of these results in the analysis of Adler-Gel'fand-Dikii flows that arise in inverse scattering and solitons is explained.
\end{abstract}

\section{Introduction.}

The differential invariants associated with a transformation group acting on a manifold are the fundamental building blocks for understanding the geometry, equivalence, symmetry and other properties of submanifolds. Moreover, the construction of general invariant differential equations and invariant variational problems requires knowledge of the differential invariants. The basic theory of differential invariants dates back to the work of Lie, [18] and Tresse, [23]. However, a complete classification of differential invariants for many of the fundamental transformation groups of physical and geometrical importance remains undeveloped. In this paper we find complete systems of differential invariants for a particularly interesting example, that of surfaces in real projective space.

The classical approach to differential invariants is via the infinitesimal methods pioneered by Lie. The main difficulty in applying Lie's method to complicated examples is that it requires the integration of linear partial differential equations, which can prove to be rather complicated. Cartan, $[3,4]$, demonstrated how his moving frame method could produce the differential invariants for several groups of geometrical interest, including the geometry of curves in the Euclidean, affine, and projective planes; see also [15]. More recently, the moving frame method was been successfully applied to study the invariants of curves in projective spaces and Grassmannians, [14]. However, extensions to more general examples has proved to be more problematic.

\footnotetext{
${ }^{1}$ Supported in part by NSF Grant DMS 98-03154.
} 
A new, practical approach to the method of moving frames was recently developed by Fels and Olver, [7, 8]. The method enables one to algorithmically implement both the practical and theoretical construction of moving frames for general transformation groups, bypassing many of the complications inherent in traditional approaches by completely avoiding the usual process of normalization during the general computation. Once a moving frame and coframe, along with the complete system of invariants, are constructed in the regularized framework, one can easily restrict these invariants to particular classes of submanifolds, producing (in nonsingular cases) the standard moving frame. Moreover, the new moving frame method provides a general mechanism for classifying differential invariants, their syzygies and commutation formulae. We are therefore able to completely describe the differential invariants, and their syzygies, for the case under consideration parametrized surfaces in $\mathbb{R} \mathbb{P}^{n-1}$.

It is worth mentioning the classical work of Fubini, [10] and Cartan, [5], on the deformations of hypersurfaces in projective space. Two surfaces are called "applicable" if they have second order contact at a point, whereas we are interested in the full equivalence problem. Cartan applies his equivalence theory of Pfaffian systems to study the particular case of surfaces in projective 3-space in detail. Cartan allows reparametrizations of the surfaces, and so his intrinsic invariants (which, unlike ours, are not explicitly written down) are different.

The theory of differential invariants has an intimate connection with the Korteweg-deVries (KdV) evolution and its generalizations, the AdlerGel'fand-Dikii (AGD) brackets. AGD brackets were defined by Adler, [2], in an attempt to extend the biHamiltonian integrability character of the $\mathrm{KdV}$ equation to generalized higher dimensional KdV evolutions. Jacobi's identity for these brackets was proved by Gel'fand and Dikii, [11]. Alternative definitions were offered later on by Kupershmidt and Wilson, [17], and by Drinfel'd and Sokolov, [6].

Consider nondegenerate parametrized projective curves in $\mathbb{R} \mathbb{P}^{n-1}$. There exists a unique way to associate to such a curve $\gamma$ a scalar differential operator $L_{\gamma}$ - a Lax operator - such that the curve is the projectivization of a solution curve of the operator, cf. [24], and such that the $(n-1)^{\text {st }}$ order term of $L_{\gamma}$ vanishes. If $\gamma$ has a monodromy, the coefficients of $L_{\gamma}$ will be periodic. The $n-1$ coefficients of the differential operator associated to the curve form a generating system of differential invariants for parametrized projective curves associated to the projective action of SL(n), [24].

Now, one can use the theory of differential invariance to write an explicit formula for the most general invariant evolution of projective curves, 
invariant under the projective action of SL(n), cf. [12]. Naturally, there will be an evolution induced upon the differential invariants themselves by this evolution of curves, and hence one obtains an evolution of Lax operators. Under certain natural conditions, this evolution was conjectured to be the AGD evolution in [12]. The lower dimensional cases (up to $n=7$ ) were also proved in [12], and the final result finally shown in [20]. KdV evolutions are obtained when special evolutions of curves are chosen. Thus, AGD evolutions can be simply described as the evolution of a generating system of differential invariants of projective curves, whenever the curves are evolving invariantly.

One can consider the problem of generalizing AGD flows to the case of two independent variables. This is an important problem not only in the theory of integrable systems, but also in related topological and algebraic areas. It seems natural to look for these generalizations among evolutions induced upon systems of differential invariants for parametrized surfaces in $\mathbb{R} \mathbb{P}^{n-1}$, whenever the surfaces themselves evolve following general invariant evolutions (invariant under the $\mathbb{R} \mathbb{P}^{n-1}$ action). A system of such differential invariants is found explicitly in this paper. Further details about AGD generalizations are given in the conclusions section.

\section{Differential Invariants.}

We begin by reviewing the basic theory of prolonged transformation groups and differential invariants. Let $M$ be an $m$-dimensional manifold. We shall consider $p$-dimensional submanifolds parametrized by immersions $\iota: X \rightarrow$ $M$, where $X$ is a fixed parameter space, which, since we are only interested in local issues, can be taken to be an open subset of $\mathbb{R}^{p}$.

Let $G$ be an $r$-dimensional Lie group acting smoothly on $M$. In particular, we are assuming that $G$ does not affect the parameters $x \in X$. Let $G_{S}=\{g \in G \mid g \cdot S=S\}$ denote the isotropy subgroup of a subset $S \subset M$, and $G_{S}^{*}=\bigcap_{x \in S} G_{x}$ its global isotropy subgroup. We assume that $G$ acts effectively on subsets of $M$, which means that $G_{U}^{*}=\{e\}$ for every open $U \subset M$. If an analytic transformation group acts effectively, it automatically acts effectively on subsets, but this equivalence does not hold in the smooth category. We say that $G$ acts freely if $G_{u}=\{e\}$ for all $u \in M$. We further incorporate the adjective "locally" in these concepts by replacing $\{e\}$ by a general discrete subgroup of $G$.

Let $\mathrm{J}^{n}=\mathrm{J}^{n}(X, M)$ denote the $n^{\text {th }}$ order jet bundle consisting of equivalence classes of submanifolds modulo $n^{\text {th }}$ order contact. We introduce local 
coordinates $x=\left(x^{1}, \ldots, x^{p}\right)$ on $X$, and $u=\left(u^{1}, \ldots, u^{q}\right)$ on $M$. The induced local coordinates on $\mathrm{J}^{n}$ are denoted by $u^{(n)}$, with components $u_{J}^{\alpha}$, where $J=\left(j_{1}, \ldots, j_{k}\right), 1 \leq j_{\nu} \leq p, 0 \leq k \leq n, \alpha=1, \ldots, q$, representing the partial derivatives of the dependent variables with respect to the independent variables. Note that

$$
\operatorname{dim} \mathrm{J}^{n}=q^{(n)}=q\left(\begin{array}{c}
p+n \\
n
\end{array}\right) .
$$

Since $G$ preserves the order of contact between submanifolds, there is an induced action of $G$ on the jet bundle $\mathrm{J}^{n}$ known as its $n^{\text {th }}$, prolongation, and denoted by $G^{(n)}$.

Definition 2.1. An $n^{\text {th }}$ order differential invariant is a function $I: \mathrm{J}^{n} \rightarrow \mathbb{R}$ which is invariant under the action of $G^{(n)}$.

Let $s_{n}$ denote the maximal orbit dimension of the prolonged action $G^{(n)}$ on $\mathrm{J}^{n}$. The stable orbit dimension is $s=\max s_{n}$. The stabilization order of $G$ is the minimal $n$ such that $s_{n}=s$. The regular subset $\mathcal{V}^{n} \subset \mathrm{J}^{n}$ is the open subset consisting of all prolonged group orbits of dimension equal to the stable orbit dimension, while the singular subset is $\mathcal{S}^{n}=\mathrm{J}^{n} \backslash \mathcal{V}^{n}$. Note that, by this definition, $\mathcal{V}^{n}=\emptyset$ and $\mathcal{S}^{n}=\mathrm{J}^{n}$ if $n$ is less than the stabilization order of $G$. If $G$ acts locally effectively on subsets, then the stabilization theorem, [21, Theorem 5.11], [22], states that $s=r=\operatorname{dim} G$, which means that $G^{(n)}$ acts locally freely on $\mathcal{V}^{n}$ for all $n$.

Proposition 2.2. In a neighborhood of any regular jet $u^{(n)} \in \mathcal{V}^{n}$, there exist $q^{(n)}-s$ functionally independent differential invariants of order at most $n$.

The traditional method for computing higher order differential invariants is via the method of invariant differentiation. In the present situation, since $G$ does not transform the parameters, the invariant differential operators are particularly simple. Namely, the parametric total derivative operators $D_{i}=D_{x^{i}}, i=1, \ldots, p$, map differential invariants to differential invariants.

Proposition 2.3. If $I\left(u^{(n)}\right)$ is any differential invariant, so are its total derivatives $D_{J} I=D_{j_{1}} \cdots D_{j_{k}} I$, where $1 \leq j_{\nu} \leq p, k=\# J \geq 0$.

Definition 2.4. A generating set of differential invariants is a finite collection $I_{1}, \ldots, I_{N}$ with the property that, for all $n$, every differential invariant (on an appropriate subset of $\mathcal{V}^{n}$ ) can be written as a function of the derivatives $D_{J} I_{\nu}$ of the generating differential invariants. 
Every transformation group admits a generating system of differential invariants. The order of a generating system can be taken to be $n+1$, where $n$ is the stabilization order. The minimal order and minimal number of differential invariants required to form a generating system is not known in general, except in the particular case of curves, $p=1$, [21].

Each of the preceding constructions has an infinitesimal counterpart. We choose a basis

$$
\mathbf{v}_{\kappa}=\sum_{\alpha=1}^{q} \varphi_{\kappa}^{\alpha}(u) \frac{\partial}{\partial u^{\alpha}}, \quad \kappa=1, \ldots, r
$$

for the Lie algebra $\mathfrak{g}$ of infinitesimal generators on $M$. Let

$$
\left\{\mathrm{pr}^{(n)} \mathbf{v}_{1}, \ldots, \operatorname{pr}^{(n)} \mathbf{v}_{r}\right\}
$$

denote the corresponding infinitesimal generators for the prolonged group action $G^{(n)}$. The prolonged generator $\mathrm{pr}^{(n)} \mathbf{v}_{\kappa}$ is obtained by truncating the infinitely prolonged vector fields

$$
\operatorname{pr} \mathbf{v}_{\kappa}=\sum_{\alpha=1}^{q} \sum_{k=\# J \geq 0} D_{J} \varphi_{\kappa}^{\alpha}\left(u^{(k)}\right) \frac{\partial}{\partial u_{J}^{\alpha}}
$$

at order $n$. The dimension of the orbit passing through $u^{(n)} \in \mathrm{J}^{n}$ equals the dimension of the subspace of $\left.T \mathrm{~J}^{n}\right|_{u^{(n)}}$ spanned by $\operatorname{pr}^{(n)} \mathbf{v}_{1}, \ldots, \operatorname{pr}^{(n)} \mathbf{v}_{r}$. In particular, if $G$ acts effectively on subsets, a jet $u^{(n)}$ is regular if and only if the vector fields $\operatorname{pr}^{(n)} \mathbf{v}_{1}, \ldots, \operatorname{pr}^{(n)} \mathbf{v}_{r}$ are linearly independent there. The infinitesimal invariance criteria are standard, [21].

Proposition 2.5. A function $I: \mathrm{J}^{n} \rightarrow \mathbb{R}$ is a differential invariant if and only if it is annihilated by the infinitesimal generators: $\operatorname{pr} \mathbf{v}_{\kappa}(I)=0, \kappa=$ $1, \ldots, r$.

\section{The Regularized Moving Frame Method.}

We now describe how to implement the regularized moving frame method in the particular case of parametrized submanifolds of an $m$-dimensional manifold $M$. Let $g=\left(g^{1}, \ldots, g^{r}\right)$ be local coordinates on $G$ in a neighborhood of the identity. Let us write out the group transformations $v=g \cdot u$ in local coordinates

$$
v^{\alpha}=\Phi^{\alpha}\left(g^{1}, \ldots, g^{r}, u^{1}, \ldots, u^{m}\right) .
$$


The functions $v^{\alpha}$ in (3.1) are referred to as the zero ${ }^{\text {th }}$ order lifted invariants, since they are invariant under the simultaneous action $(h, u) \mapsto\left(h \cdot g^{-1}, g \cdot u\right)$ of $G$ on the trivial principal bundle $G \times M$. Since $G$ does not act on the parameters, the corresponding prolonged transformations $v^{(n)}=g^{(n)} \cdot u^{(n)}$ are easily obtained by total differentiation. The resulting functions $v_{J}^{\alpha}=$ $D_{J} v^{\alpha}$ are called the lifted differential invariants since they are invariant under the simultaneous action $\left(h, u^{(n)}\right) \rightarrow\left(h \cdot g^{-1}, g^{(n)} \cdot u^{(n)}\right)$ on $G \times \mathrm{J}^{n}$.

The primary use of a moving frame is that it enables one to pass from lifted invariant objects, which are trivial, to their ordinary invariant counterparts back on the original manifold and its jet spaces. This allows us to systematically analyze the invariants via the particularities of the moving frame. The following fundamental definition appears in [8], and is motivated by earlier work of Griffiths, [14], and Jensen, [16].

Definition 3.1. An $n^{\text {th }}$ order moving frame is a map $\rho^{(n)}: \mathrm{J}^{n} \rightarrow G$ which is (locally) $G$-equivariant with respect to the prolonged action $G^{(n)}$ on $\mathrm{J}^{n}$, and the right action $h \mapsto h \cdot g^{-1}$ of $G$ on itself.

Remark. For simplicity, we shall only consider right moving frames in this paper. A left moving frame, which is equivariant with respect to left multiplication $h \mapsto g \cdot h$ is easily obtained by inverting the right moving frame: $\rho^{(n)}(g)^{-1}$.

Theorem 3.2. If $G$ acts effectively on subsets, then an $n^{\text {th }}$ order moving frame exists in a neighborhood of a point $u^{(n)} \in \mathrm{J}^{n}$ if and only if $u^{(n)} \in \mathcal{V}^{n}$ is a regular jet.

In particular, the minimal order at which any moving frame exists is the stabilization order of the group. In practical implementations, the normalization procedure for constructing moving frames amounts to choosing a (local) cross-section $\mathcal{K}^{n} \subset \mathcal{V}^{n}$ to the (regular) prolonged group orbits. In other words, $\mathcal{K}^{n}$ is a submanifold of dimension $q^{(n)}-r$ which intersects each orbit at most once, and transversally. Given $u^{(n)} \in \mathcal{V}^{n}$, let $g=\rho^{(n)}\left(u^{(n)}\right)$ denote the group element that maps $u^{(n)}$ to the cross-section:

$$
g^{(n)} \cdot u^{(n)}=\rho^{(n)}\left(u^{(n)}\right) \cdot u^{(n)} \in \mathcal{K}^{n} .
$$

The resulting map $\rho^{(n)}: \mathrm{J}^{n} \rightarrow G$ is a moving frame. Moreover, every moving frame has this form, where the cross-section equals the preimage $\mathcal{K}^{n}=$ $\left(\rho^{(n)}\right)^{-1}\{e\}$ of the identity element. 
The simplest local cross-sections are obtained by setting $r=\operatorname{dim} G$ of the jet coordinates $u^{(n)}$ to be constant. We denote the chosen coordinates by $u_{\nu} \equiv u_{J_{\nu}}^{\alpha_{\nu}}, \nu=1, \ldots, r$. Therefore, $\mathcal{K}^{n}=\left\{u_{1}=c_{1}, \ldots, u_{r}=c_{r}\right\}$, where the normalization constants $c_{1}, \ldots, c_{r}$ are chosen so that the normalization equations (3.2), which have the form

$$
v_{1}=v_{J_{1}}^{\alpha_{1}}\left(g, u^{(n)}\right)=c_{1}, \quad \ldots \quad v_{r}=v_{J_{r}}^{\alpha_{r}}\left(g, u^{(n)}\right)=c_{r},
$$

can be (locally) uniquely solved for $g=\rho^{(n)}\left(u^{(n)}\right)$ in terms of the jet coordinates. The resulting map defines the moving frame associated with the chosen cross-section.

Remark 1. Any $n^{\text {th }}$ order moving frame $\rho^{(n)}: \mathrm{J}^{n} \rightarrow G$ can also be viewed as a moving frame of any higher order $k \geq n$ by composing with the standard jet space projection $\pi_{n}^{k}: \mathrm{J}^{n} \rightarrow \mathrm{J}^{k}$. In the sequel, we will speak of "moving frames of order $n$ " with the understanding that they may very well have been constructed at some lower order.

We now describe how the moving frame provides us with a complete system of differential invariants.

Definition 3.3. The fundamental $n^{\text {th }}$ order normalized differential invariants associated with a moving frame $\rho^{(n)}$ of order $n$ or less are given by

$$
I^{(n)}\left(u^{(n)}\right)=v^{(n)}\left(\rho^{(n)}\left(u^{(n)}\right), u^{(n)}\right)=\rho^{(n)}\left(u^{(n)}\right) \cdot u^{(n)} .
$$

In other words, the individual components of $I^{(n)}$, which are

$$
I_{K}^{\alpha}\left(u^{(k)}\right)=v_{K}^{\alpha}\left(\rho^{(n)}\left(u^{(n)}\right), u^{(k)}\right), \quad \alpha=1, \ldots, q, \quad k=\# K \geq 0,
$$

define differential invariants of order $\leq n$. Note that the normalized differential invariants corresponding to the components being normalized via (3.3) will be constant. We shall call these the phantom differential invariants. The other components of $v^{(n)}$ will define a complete system of functionally independent differential invariants defined on the domain of definition of the moving frame map. This will hold for any order $n$ at least as large as the order of the chosen moving frame.

Theorem 3.4. Let $n$ be greater than or equal to the order of the moving frame. Every $n^{\text {th }}$ order differential invariant can be locally written as a function of the normalized $n^{\text {th }}$ order differential invariants $I^{(n)}$. The function is unique provided it does not depend on the phantom invariants. 
A moving frame therefore provides a natural way to construct a differential invariant from any differential function.

Definition 3.5. The invariantization with respect to the given moving frame of a differential function $F: \mathrm{J}^{n} \rightarrow \mathbb{R}$ is the differential invariant $F \circ I^{(n)}$.

In particular, if $F$ is itself a differential invariant, then it coincides with its invariantization: $F=F \circ I^{(n)}$. Thus, invariantization defines a projection, depending on the moving frame, from the space of differential functions to the space of differential invariants.

An alternative method to construct higher order differential invariants is by invariant differentiation, as in Proposition 2.3. A critical remark, however, is that the total derivative of a normalized differential invariant is not necessarily equal to the corresponding higher order normalized differential invariant. The fundamental recurrence formulae for the differential invariants (3.5) are

$$
D_{j} I_{K}^{\alpha}=I_{K, j}^{\alpha}+M_{K ; j}^{\alpha}
$$

Higher order versions,

$$
D_{J} I_{K}^{\alpha}=I_{K, J}^{\alpha}+M_{K ; J}^{\alpha}
$$

are obtained by differentiating (3.6). For example,

$$
D_{k} D_{j} I_{K}^{\alpha}=D_{k} I_{K, j}^{\alpha}+D_{k} M_{K ; j}^{\alpha}=I_{K, j, k}^{\alpha}+\sum_{\beta, L} \frac{\partial M_{K ; j}^{\alpha}}{\partial I_{L}^{\beta}}\left(I_{L, k}^{\beta}+M_{L ; k}^{\beta}\right) .
$$

Remark. While $I_{K, J}^{\alpha}$ is symmetric under permutations of the multi-index $(K, J)$, this is not true for $M_{K ; J}^{\alpha}$, which is why we use a semicolon to separate the two indices.

The "correction terms" $M_{K ; j}^{\alpha}$ can be explicitly computed using the following algorithm, which is justified in [9]. Let $V=V^{(n)}$ denote the $r \times q^{(n)}$ matrix whose entries are the coefficients $D_{J} \varphi_{\kappa}^{\alpha}$ of the $n^{\text {th }}$ order prolonged infinitesimal generators (2.3). Let $W=V \circ I^{(n)}$ be its invariantization, obtained by replacing the jet coordinates $u^{(n)}$ by the associated normalized differential invariants $I^{(n)}$. We perform a Gauss-Jordan row reduction on the matrix $W$ so as to reduce the $r \times r$ minor whose columns correspond to the chosen normalization variables $u_{1}, \ldots, u_{r}$ to be the identity matrix; 
let $P$ be the resulting $r \times q^{(n)}$ matrix of invariants. Let $S=\left(S_{i}^{\kappa}\right)$ denote the $p \times r$ matrix whose entries are the total derivatives $S_{i}^{\kappa}=D_{i} u_{\kappa}$ of the normalization coordinates. Let $T=S \circ I^{(n)}$ be its invariantization. Then the correction terms in (3.6) are the entries of the $p \times q^{(n)}$ matrix product $M=-T \cdot P$.

Except in the case of curves, where $p=1$, the differentiated invariants are not necessarily functionally independent. (Again, this is not the case for the non-constant normalized differential invariants.) A syzygy is a functional dependency $H\left(\ldots \mathcal{D}_{J} I_{\nu} \ldots\right) \equiv 0$ among the fundamental differentiated invariants. The recurrence formulae not only provide us with a generating system of fundamental differential invariants, but also classify all syzygies among the normalized differential invariants.

Theorem 3.6. A generating system of differential invariants consists of

a) all non-phantom zero ${ }^{\text {th }}$ order differential invariants $I^{\alpha}$, and

b) all non-phantom differential invariants of the form $I_{J, i}^{\alpha}$ where $I_{J}^{\alpha}$ is a phantom differential invariant.

In other words, every other differential invariant can, locally, be written as a function of the generating invariants and their invariant derivatives, $D_{K} I_{J, i}^{\alpha}$.

All syzygies among the differentiated invariants are differential consequences of the following two fundamental types:

(i) $D_{J} I_{K}^{\alpha}=c_{\nu}+M_{K, J}^{\alpha}$, when $I_{K}^{\alpha}$ is a generating differential invariant, while $I_{J, K}^{\alpha}=c_{\nu}$ is a phantom differential invariant, and

(ii) $D_{J} I_{L K}^{\alpha}-\mathcal{D}_{K} I_{L J}^{\alpha}=M_{L K ; J}^{\alpha}-M_{L J ; K}^{\alpha}$, where $I_{L K}^{\alpha}$ and $I_{L J}^{\alpha}$ are generating differential invariants, the multi-indices $K \cap J=\emptyset$ are disjoint and non-zero, while $L$ is an arbitrary multi-index.

A minimal system of differential invariants can be found by a careful analysis of the recurrence relations and consequent syzygies. Examples appear in [8] and below.

\section{Equivalence, Symmetry and Rigidity.}

Classically, in geometrical applications, a moving frame is defined on a single submanifold of the underlying space. Indeed, in applications to equivalence problems and symmetry, we restrict the moving frame and associated invariants to a submanifold of the appropriate dimension. 
Definition 4.1. A $p$-dimensional submanifold parametrized by $\iota: X \rightarrow$ $S \subset M$ is called regular with respect to a moving frame $\rho^{(n)}: \mathrm{J}^{n} \rightarrow G$ if its $n$-jet $\mathrm{j}_{n} S$ lies in the domain of definition of $\rho^{(n)}$. In this case, the restricted moving frame on the submanifold is defined as the composition $\rho^{(n)} \circ \mathrm{j}_{n} \iota: X \rightarrow G$.

Theorem 4.2. A submanifold $S \subset M$ (locally) admits an $n^{\text {th }}$ order moving frame if and only if $S$ is regular of order n, i.e. $\mathrm{j}_{n} S \subset \mathcal{V}^{n}$. In the analytic category, $S$ admits a moving frame (of some sufficiently high order) if and only if $G_{S}$ acts locally freely on $S$.

Let $S$ be a regular submanifold for a moving frame $\rho^{(n)}$. For any $k \geq n$, the $k^{\text {th }}$ order differential invariant classifying manifold $\mathcal{C}^{(k)}(S)$ associated with a submanifold $\iota: X \rightarrow M$ is the manifold parametrized by the normalized differential invariants of order $k$, namely $J^{(k)}=I^{(k)} \circ j_{k} \iota$. For simplicity, let us assume that, for each $k \geq n, \mathcal{C}^{(k)}(S)$ is an embedded submanifold of its classifying space $Z^{(k)} \simeq \mathrm{J}^{k}$. Note that $t_{k}=\operatorname{dim} \mathcal{C}^{(k)}(S)$ equals the number of functionally independent invariants obtained by restricting the normalized $k^{\text {th }}$ order differential invariants to $S$. In the fully regular case, then, we have

$$
t_{n}<t_{n+1}<t_{n+2}<\cdots<t_{s}=t_{s+1}=\cdots=t \leq p
$$

where $t$ is called the differential invariant rank and $s$ is the differential invariant order of $S$. We can now state the fundamental equivalence and symmetry theorems.

Theorem 4.3. Let $S, \bar{S} \subset M$ be regular $p$-dimensional submanifolds with respect to a moving frame map $\rho^{(n)}$. Then $S$ and $\bar{S}$ are (locally) congruent, $\bar{S}=g \cdot S$, if and only if they have the same differential invariant order $s$, and their classifying manifolds of order $s+1$ are identical: $\mathcal{C}^{(s+1)}(\bar{S})=\mathcal{C}^{(s+1)}(S)$.

Theorem 4.4. Let $S \subset M$ be a regular p-dimensional submanifold of differential invariant rank $t$ with respect to a moving frame $\rho^{(n)}$. Then its isotropy group $G_{S}$ is an $(r-t)$-dimensional subgroup of $G$ acting locally freely on $S$.

A submanifold $S$ is order $k$ congruent to a submanifold $\bar{S}$ at $z \in S$ if there is a group transformation $g \in G$ such that $S$ and $g \cdot \bar{S}$ have order $k$ contact at $z$. Note that the group transformation $g=g(z)$ may vary from point to point. The rigidity order of $S$ is the minimal $k$ for which order $k$ congruence implies congruence, so $\bar{S}=g \cdot S$ for fixed $g \in G$; see [14, 13]. It 
turns out that this also means that the only congruent submanifold $\bar{S}=g \cdot S$ which has $k^{\text {th }}$ order contact with $S$ at a point is $S$ itself.

Theorem 4.5. If $S \subset M$ is a regular submanifold of differential invariant order $s$ with respect to a moving frame, then $S$ has rigidity order at most $s+1$.

This completes our review of the general theory of moving frames. We now apply these techniques to the problem at hand - differential invariants for surfaces in projective space under the action of the projective group.

\section{Notation.}

We will apply the normalized moving frame method described above to the case of parametrized surfaces in $\mathbb{R P}^{n-1}$, under the projective action of the unimodular group SL $(n)$. Thus we shall consider immersions $u: X \rightarrow$ $\mathbb{R} \mathbb{P}^{n-1}$, where $X$ is a two-dimensional parameter space. We shall use local coordinates $x, y$ on $X$, and a standard set of projective coordinates $u=$ $\left(u^{1}, \ldots, u^{n-1}\right)$ on $\mathbb{R} \mathbb{P}^{n-1}$. In other words, we have two independent variables and $n-1$ dependent variables.

Let $\mathrm{J}^{n}=\mathrm{J}^{n}\left(X, \mathbb{R} \mathbb{P}^{n-1}\right)$ denote the $n^{\text {th }}$ order jet bundle for surfaces in $\mathbb{R} \mathbb{P}^{n-1}$. The local derivative coordinates $u^{(n)}$ on $\mathrm{J}^{n}$ are given by $u_{i j}^{\alpha}=$ $D_{x}^{i} D_{y}^{j} u^{\alpha}$. The order of the derivative is $m=i+j$. Low order derivatives will be denoted by $u_{x}^{\alpha}=u_{1,0}^{\alpha}=D_{x} u^{\alpha}, u_{x x}^{\alpha}=u_{2,0}^{\alpha}=D_{x}^{2} u^{\alpha}, u_{x y}^{\alpha}=u_{1,1}^{\alpha}=$ $D_{x} D_{y} u^{\alpha}$, etc. We shall introduce the natural degree lexicographic ordering on the derivative multi-indices $(i, j)$, so that the first few are ordered as follows:

$$
(0,0),(1,0), \quad(0,1),(2,0),(1,1),(0,2),(3,0), \ldots .
$$

More specifically, the map

$$
k=\lambda(i, j)=\frac{1}{2}(i+j)(i+j+1)+j,
$$

defines a bijection $\lambda: \mathbb{N}^{2} \stackrel{\sim}{\longrightarrow} \mathbb{N}$, such that $(i, j)$ is the $k=\lambda(i, j)^{\text {th }}$ index in the lexicographic ordering. We use the convenient alternative notations

$$
\widetilde{D}_{k}=D_{x}^{i} D_{y}^{j}, \quad \text { and } \quad \tilde{u}_{k}^{\alpha}=u_{i j}^{\alpha}, \quad \text { when } \quad k=\lambda(i, j),
$$

for derivatives and differential operators. In particular,

$$
\begin{array}{lll}
\tilde{u}_{1}^{\alpha}=u_{0,0}^{\alpha}=u^{\alpha}, & \tilde{u}_{2}^{\alpha}=u_{1,0}^{\alpha}=u_{x}^{\alpha}, & \tilde{u}_{3}^{\alpha}=u_{0,1}^{\alpha}=u_{y}^{\alpha}, \\
\tilde{u}_{4}^{\alpha}=u_{2,0}^{\alpha}=u_{x x}^{\alpha}, & \tilde{u}_{5}^{\alpha}=u_{1,1}^{\alpha}=u_{x y}^{\alpha}, & \tilde{u}_{6}^{\alpha}=u_{0,2}^{\alpha}=u_{y y}^{\alpha},
\end{array}
$$


and so on. Given $k=\lambda(i, j)$, we define $\mu(k)=m=i+j$, which is the order of the derivative $\widetilde{D}_{k}$ corresponding to $k$. It equals the smallest triangular number $t_{m}=\frac{1}{2} m(m+1) \leq k$. Define the map $\sigma: \mathbb{N} \times \mathbb{N} \rightarrow \mathbb{N}$ so that given $k=\lambda(i, j)$ and $l=\lambda\left(i^{\prime}, j^{\prime}\right)$, we set $p=\sigma(k, l)=\lambda\left(i+i^{\prime}, j+j^{\prime}\right)$. Therefore,

$$
\widetilde{D}_{\sigma(k, l)}=\widetilde{D}_{k} \cdot \widetilde{D}_{l}, \quad \text { and } \quad \tilde{u}_{\sigma(k, l)}^{\alpha}=\widetilde{D}_{k}\left(\tilde{u}_{l}^{\alpha}\right)=\widetilde{D}_{l}\left(\tilde{u}_{k}^{\alpha}\right) .
$$

Further, define $q=\tau(k, l)=\lambda\left(i-i^{\prime}, j-j^{\prime}\right)$. Note that $\tau(k, l)$ is defined if and only if $i^{\prime} \leq i$ and $j^{\prime} \leq j$. We further define

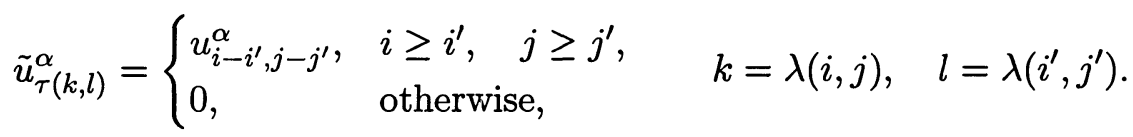

Finally, define the binomial coefficients

$$
\llbracket \begin{aligned}
& k \\
& l
\end{aligned} \rrbracket=\left\{\begin{array}{l}
\left(\begin{array}{c}
i \\
i^{\prime}
\end{array}\right)\left(\begin{array}{l}
j \\
j^{\prime}
\end{array}\right), \quad i \geq i^{\prime}, \quad j \geq j^{\prime} \\
0, \quad \text { otherwise, }
\end{array} \quad k=\lambda(i, j), \quad l=\lambda\left(i^{\prime}, j^{\prime}\right) .\right.
$$

Consider the projective action of $\operatorname{SL}(n)$ on $\mathbb{R} \mathbb{P}^{n-1}$. Given a unimodular matrix $A=\left(a_{j}^{i}\right)$, where $i, j=1, \ldots, n$, the associated transformation rules are given by

$$
v^{\alpha}=\frac{\sum_{\beta=1}^{n-1} a_{\beta}^{\alpha} u^{\beta}+a_{n}^{\alpha}}{\sum_{\beta=1}^{n-1} a_{\beta}^{n} u^{\beta}+a_{n}^{n}}, \quad \alpha=1, \ldots, n-1
$$

The components of $v$ in (5.6) are the lifted invariants of order zero: $v=$ $A \cdot u$. Since we are dealing with parametric surfaces, the higher order lifted invariants, which are the components of the prolonged group action $v^{(n)}=$ $A \cdot u^{(n)}$, are given by differentiation:

$$
\tilde{v}_{k}^{\alpha}=\tilde{v}_{i j}^{\alpha}=D_{x}^{i} D_{y}^{j} v^{\alpha}=\widetilde{D}_{k} v^{\alpha}, \quad \text { when } \quad k=\lambda(i, j)
$$

The infinitesimal generators are given by

$$
\mathbf{v}_{\alpha}=\frac{\partial}{\partial u^{\alpha}}, \quad \mathbf{v}_{\alpha \beta}=u^{\alpha} \frac{\partial}{\partial u^{\beta}}, \quad \mathbf{w}_{\alpha}=u^{\alpha} \mathbf{s}, \quad \alpha, \beta=1, \ldots, n-1 .
$$

Here

$$
\mathbf{s}=\sum_{\beta=1}^{3} u^{\beta} \frac{\partial}{\partial u^{\beta}}=\sum_{\beta=1}^{3} \mathbf{v}_{\beta \beta}
$$

is the scaling vector field. 


\section{The case of surfaces in $\mathbb{R} \mathbb{P}^{3}$.}

In this section we will describe in detail the simplest case, which is that of surfaces in $\mathbb{R P}^{3}$. The results and computations will serve as an illustration of what we expect to find in the general situation.

Consider a parametrized surface $u(x, y)=\left(u^{1}(x, y), u^{2}(x, y), u^{3}(x, y)\right) \in$ $\mathbb{R P}^{3}$. Given three multi-indices $k=\lambda(i, j), l=\lambda\left(i^{\prime}, j^{\prime}\right), m=\lambda\left(i^{\prime \prime}, j^{\prime \prime}\right)$, we define the associated $3 \times 3$ matrix of partial derivatives

$$
\widetilde{U}_{3}(k, l, m)=U_{3}\left(i j ; i^{\prime} j^{\prime} ; i^{\prime \prime} j^{\prime \prime}\right)=\left(\begin{array}{ccc}
u_{i j}^{1} & u_{i^{\prime} j^{\prime}}^{1} & u_{i^{\prime \prime} j^{\prime \prime}}^{1} \\
u_{i j}^{2} & u_{i^{\prime} j^{\prime}}^{2} & u_{i^{\prime \prime} j^{\prime \prime}}^{2} \\
u_{i j}^{3} & u_{i^{\prime} j^{\prime}}^{3} & u_{i^{\prime \prime} j^{\prime \prime}}^{3}
\end{array}\right)=\left(\begin{array}{ccc}
\tilde{u}_{k}^{1} & \tilde{u}_{l}^{1} & \tilde{u}_{m}^{1} \\
\tilde{u}_{k}^{2} & \tilde{u}_{l}^{2} & \tilde{u}_{m}^{2} \\
\tilde{u}_{k}^{3} & \tilde{u}_{l}^{3} & \tilde{u}_{m}^{3}
\end{array}\right)
$$

Let

$$
\Delta_{3}(k, l, m)=\operatorname{det} \widetilde{U}_{3}(k, l, m)
$$

denote its determinant. In low order cases, we can replace $(i, j)$, etc., by derivatives indices. The most important of these is

$$
U_{3}^{*}=\widetilde{U}_{3}(1,2,3)=U_{3}(x, y, x x)=\left(\begin{array}{ccc}
u_{x}^{1} & u_{y}^{1} & u_{x x}^{1} \\
u_{x}^{2} & u_{y}^{2} & u_{x x}^{2} \\
u_{x}^{3} & u_{y}^{3} & u_{x x}^{3}
\end{array}\right)
$$

with determinant $\Delta_{3}^{*}=\operatorname{det} U_{3}^{*}$.

Definition 6.1. A parametrized surface is nondegenerate if the matrix (6.2) is nonsingular: $\Delta_{3}^{*}=\operatorname{det} U_{3}(x, y, x x) \neq 0$.

Actually, we shall assume a "convexity" condition that $\Delta_{3}^{*}>0$. Note that the sign of $\Delta_{3}^{*}$ can be switched by relabeling the coordinates in $\mathbb{R P}^{3}$.

Remark. We have chosen this particular nondegeneracy condition just for specificity. We could, alternatively, replace the second derivative with respect to $x$ by either of the other second order derivatives.

We now begin our implementation of the normalization procedure. The first step is to normalize the components $v^{1}=v^{2}=v^{3}=0$ of the order zero invariants (5.6) by solving for some of the parameters $a_{j}^{i}$. This leads us to the choice

$$
a_{4}^{\alpha}=-\sum_{\beta=1}^{3} a_{\beta}^{\alpha} u^{\beta}, \quad \alpha=1, \ldots, 3 .
$$


This normalization will generalize in an obvious way to the $n$-dimensional case.

Once we have solved for the parameters $a_{4}^{\alpha}$ as in (6.3), we next normalize the corresponding matrix consisting of first and second order lifted invariants,

$$
V_{3}^{*}=\left(\begin{array}{ccc}
v_{x}^{1} & v_{y}^{1} & v_{x x}^{1} \\
v_{x}^{2} & v_{y}^{2} & v_{x x}^{2} \\
v_{x}^{3} & v_{y}^{3} & v_{x x}^{3}
\end{array}\right)=I
$$

to be the identity matrix. Let us call

$$
K=\sum_{\beta=1}^{3} a_{\beta}^{4} u^{\beta}+a_{4}^{4} .
$$

The value of the relative invariant $K$ will be fixed later. Further, we let

$$
K_{k}=K_{i, j}=\widetilde{D}_{k} K=\sum_{\beta=1}^{3} a_{\beta}^{4} u_{i j}^{\beta} \quad \text { when } \quad k=\lambda(i, j),
$$

denote the derivatives of $K$, prior to normalization. For example $K_{x}=$ $D_{x} K=\sum_{\beta} a_{\beta}^{4} u_{x}^{\beta}$.

The formula

$$
v_{x x}^{\alpha}=\frac{1}{K}\left(\sum_{\beta=1}^{3} a_{\beta}^{\alpha} u_{x x}^{\beta}-2 v_{x}^{\alpha} \sum_{\beta=1}^{3} a_{\beta}^{4} u_{x}^{\beta}-v^{\alpha} \sum_{\beta=1}^{3} a_{\beta}^{4} u_{x x}^{\beta}\right)
$$

implies that the normalization (6.4) yields the relationship

$$
\tilde{A} \cdot U_{3}^{*}=\left(\begin{array}{ccc}
K & 0 & 2 K_{x} \\
0 & K & 0 \\
0 & 0 & K
\end{array}\right)
$$

where $\widetilde{A}$ denotes the upper $3 \times 3$ minor of the $4 \times 4$ matrix $A$. Equation (6.7) fixes the parameters in $\widetilde{A}$ in terms of $K$ and its derivatives. Namely

$$
\widetilde{A}=\left(\begin{array}{lll}
a_{1}^{1} & a_{2}^{1} & a_{3}^{1} \\
a_{1}^{2} & a_{2}^{2} & a_{3}^{2} \\
a_{1}^{3} & a_{2}^{3} & a_{3}^{3}
\end{array}\right)=\left(\begin{array}{ccc}
K & 0 & 2 K_{x} \\
0 & K & 0 \\
0 & 0 & K
\end{array}\right)\left(U_{3}^{*}\right)^{-1}
$$

The final step in the normalization process is to solve for the parameters $a_{\beta}^{4}, \beta=1, \ldots, 4$. These will require higher order normalizations that will 
produce the three terms $K_{x}, K_{y}$ and $K_{x x}$, the last one depending on third order derivatives. A particularly convenient cross-section is provided by the additional normalizations

$$
v_{x y}^{2}=v_{y y}^{2}=v_{x x y}^{2}=0 .
$$

This leads to the identities

$$
\left(K_{x}, K_{y}, K_{x x}\right)=\left(\begin{array}{lll}
a_{1}^{4} & a_{2}^{4} & a_{3}^{4}
\end{array}\right) U_{3}^{*}=\left(\begin{array}{lll}
a_{1}^{2} & a_{2}^{2} & a_{3}^{2}
\end{array}\right)\left(\begin{array}{ccc}
u_{x y}^{1} & \frac{1}{2} u_{y y}^{1} & u_{x x y}^{1} \\
u_{x y}^{2} & \frac{1}{2} u_{y y}^{2} & u_{x x y}^{2} \\
u_{x y}^{3} & \frac{1}{2} u_{y y}^{3} & u_{x x y}^{3}
\end{array}\right) .
$$

From here we are able to determine uniquely $a_{1}^{4}, a_{2}^{4}, a_{3}^{4}$, by substituting the value of $a_{\beta}^{2}$ from (6.8) and using Cramer's rule. We find

$$
\left(\begin{array}{lll}
a_{1}^{4} & a_{2}^{4} & a_{3}^{4}
\end{array}\right)=-\frac{K}{\Delta_{3}^{*}}\left(\Delta_{3}(x y), \frac{1}{2} \Delta_{3}(y y), \Delta_{3}(x x y)\right),
$$

where we abbreviate

$$
\Delta_{3}(k)=\Delta_{3}(x, x x, k) .
$$

Finally, notice that condition (6.3) implies that the determinant of $A$, after normalization, equals

$$
\operatorname{det} A=\operatorname{det}\left(\begin{array}{cccc}
a_{1}^{1} & a_{2}^{1} & a_{3}^{1} & 0 \\
a_{1}^{2} & a_{2}^{2} & a_{3}^{2} & 0 \\
a_{1}^{3} & a_{2}^{3} & a_{3}^{3} & 0 \\
a_{1}^{4} & a_{2}^{4} & a_{3}^{4} & K
\end{array}\right)=K \operatorname{det}\left(\begin{array}{ccc}
a_{1}^{1} & a_{2}^{1} & a_{3}^{1} \\
a_{1}^{2} & a_{2}^{2} & a_{3}^{2} \\
a_{1}^{3} & a_{2}^{3} & a_{3}^{3}
\end{array}\right)=\frac{K^{4}}{\Delta_{3}^{*}},
$$

using (6.8). Thus, it suffices to choose $K=\left(\Delta_{3}^{*}\right)^{-1 / 4}$ to guarantee that $A$ still belongs to SL(4). This finally fixes the value of $a_{4}^{4}$, and hence all of the rest of the parameters. This way, we obtain a third order moving frame $\rho^{(3)}: J^{3} \longrightarrow \mathrm{SL}(4)$. The explicit formula is

$$
\begin{aligned}
& \rho^{(3)}\left(u^{(3)}\right)=\left(\Delta_{3}^{*}\right)^{-\frac{5}{4}}
\end{aligned}
$$

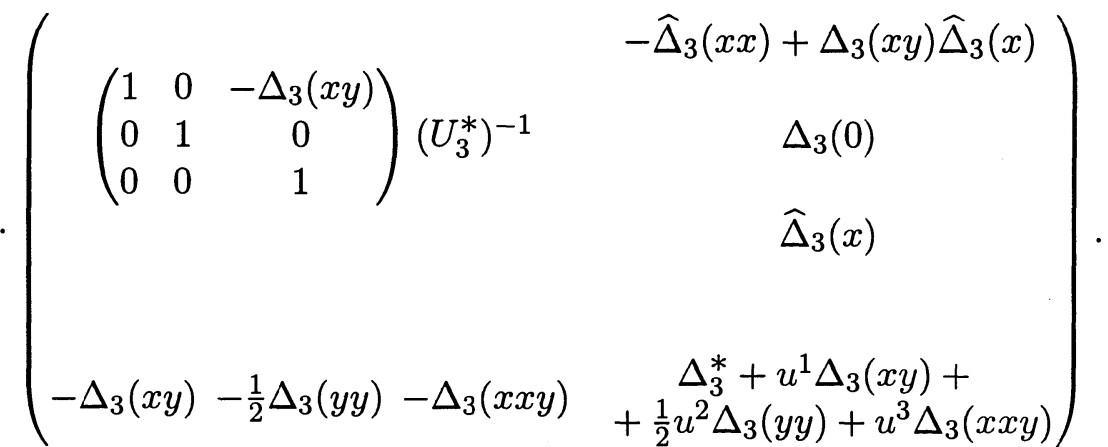


where

$$
\widehat{\Delta}_{3}(k)=\Delta_{3}(0, y, k),
$$

the 0 indicating that the first column of the determinant consists of the undifferentiated variables $\left(u^{1}, u^{2}, u^{3}\right)$.

It is not too hard to prove that this frame is of minimal order, since the $\mathrm{SL}(4)$ projective action on $\mathbb{R P}^{3}$ has stabilization order 3 . In view of (5.8), the second order prolonged vector fields $\operatorname{pr}^{(2)} \mathbf{v}_{\alpha}, \operatorname{pr}^{(2)} \mathbf{v}_{\alpha \beta}, \alpha, \beta=1,2,3$, are all linearly independent on nondegenerate jets, where $\Delta_{3}^{*} \neq 0$. In fact, we only need to consider the derivatives with respect to $x, y$, and $x x$ to achieve that condition. On the other hand, we need to go up to order three to make $\mathrm{pr}^{(k)} \mathbf{w}_{\alpha}$ linearly independent. Indeed, if $f, g$ and $h$ are functions such that

$$
f u_{x}^{1}+g u_{x}^{2}+h u_{x}^{3}=f u_{y}^{1}+g u_{y}^{2}+h u_{y}^{3}=0
$$

then it is not hard to show that $f \mathrm{pr}^{(2)} \mathbf{w}_{1}+g \operatorname{pr}^{(2)} \mathbf{w}_{2}+h \operatorname{pr}^{(2)} \mathbf{w}_{3}$ is a combination of the prolongations $\operatorname{pr}^{(2)} \mathbf{v}_{\alpha}$ and $\operatorname{pr}^{(2)} \mathbf{v}_{\alpha \beta}, \alpha, \beta=1,2,3$. This does not happen once we go up to third order, for precisely the same reason that the normalization above will bring the lowest order frame possible. Indeed, at that point $\Delta_{3}^{*} \neq 0$ so that no linear relationship between $u_{x}^{\alpha}, u_{y}^{\alpha}$ and $u_{x x}^{\alpha}$ can be found.

The description of a generating set of invariants and their associated syzygies becomes quite straightforward once a frame has been found. We simply have to turn to Theorem 3.6 where the classification was found in terms of the frame.

Let $I^{(n)}$ be the normalized differential invariants, defined in terms of our moving frame (6.10) as in (3.5). Then, according to the normalizations we used to achieve our frame, the phantom invariants will be

$$
I^{\alpha}, \quad I_{x}^{\alpha}, \quad I_{y}^{\alpha}, \quad I_{x x}^{\alpha}, \quad \alpha=1,2,3, \quad I_{x y}^{2}, \quad I_{y y}^{2}, \quad I_{x x y}^{2} .
$$

Consequently, Theorem 3.6 implies that the normalized invariants

$$
\begin{aligned}
& I_{x y}^{1}, \quad I_{y y}^{1}, \quad I_{x x x}^{1}, \quad I_{x x y}^{1}, \\
& I_{x y}^{3}, \quad I_{y y}^{3}, \quad I_{x x x}^{3}, \quad I_{x x y}^{3}, \\
& I_{x x x}^{2}, \quad I_{x y y}^{2}, \quad I_{y y y}^{2}, \quad I_{x x x y}^{2}, \quad I_{x x y y}^{2},
\end{aligned}
$$

provide a generating set of differential invariants. However, this set is far from minimal, as we shall see from the recurrence formulae (3.6).

In order to obtain the correction terms $M_{K ; j}^{\alpha}$ we proceed as described in Section 3. The matrix $T$ is obtained as follows. We first assemble the 
derivatives of the lifted invariants that were normalized to produce the frame (i.e. the ones defining the phantom invariants) into a matrix by placing the $x$ derivatives in the first and the $y$ derivatives in the second row. The invariantization of the resulting matrix, that is, replacing the jet coordinates by their associated invariant, produces $T$. In constructing these matrices, we use the following convenient order on our jet space coordinates:

$$
u^{1}, u^{2}, u^{3}, u_{x}^{1}, u_{y}^{1}, u_{x x}^{1}, u_{x}^{2}, u_{y}^{2}, u_{x x}^{2}, u_{x}^{3}, u_{y}^{3}, u_{x x}^{3}, u_{x y}^{2}, u_{y y}^{2}, u_{x x y}^{2},
$$

followed by

$$
\tilde{u}_{5}^{1}, \tilde{u}_{6}^{1}, \tilde{u}_{7}^{1}, \tilde{u}_{8}^{1}, \ldots, \tilde{u}_{7}^{2}, \tilde{u}_{9}^{2}, \tilde{u}_{10}^{2}, \ldots, \tilde{u}_{5}^{3}, \tilde{u}_{6}^{3}, \tilde{u}_{7}^{3}, \tilde{u}_{8}^{3}, \ldots
$$

Using the first part of the assigned ordering, we see that

$$
T=\left(\begin{array}{ccccccccccccccc}
1 & 0 & 0 & 0 & I_{x y}^{1} & I_{x x x}^{1} & 0 & 0 & I_{x x x}^{2} & 1 & I_{x y}^{3} & I_{x x x}^{3} & 0 & I_{x y y}^{2} & I_{x x x y}^{2} \\
0 & 1 & 0 & I_{x y}^{1} & I_{y y}^{1} & I_{x x y}^{1} & 0 & 0 & 0 & I_{x y}^{3} & I_{y y}^{3} & I_{x x y}^{3} & I_{x y y}^{2} & I_{y y y}^{2} & I_{x x y y}^{2}
\end{array}\right)
$$

The rows of the infinitesimal generator coefficient matrix $V$ will be written in the following order:

$$
\mathbf{v}_{1}, \mathbf{v}_{2}, \mathbf{v}_{3}, \mathbf{v}_{11}, \mathbf{v}_{21}, \mathbf{v}_{31}, \mathbf{v}_{12}, \mathbf{v}_{22}, \mathbf{v}_{32}, \mathbf{v}_{13}, \mathbf{v}_{23}, \mathbf{v}_{33}, \mathbf{w}_{1}, \mathbf{w}_{2}, \mathbf{w}_{3} .
$$

Once that ordering is in place, we replace each $\tilde{u}_{k}^{i}$ by the corresponding invariant $\widetilde{I}_{k}^{i}$, which gives the invariantization of $V$ as

$$
\begin{aligned}
& W= \\
& \left(\begin{array}{cccccccc}
I & 0 & 0 & 0 & 0 & 0 & 0 & 0 \\
0 & I & 0 & 0 & 0 & A_{3}(5,6,7, \ldots) & 0 & 0 \\
0 & 0 & I & 0 & A_{3}(5,6,8) & 0 & A_{3}(7,9,10, \ldots) & 0 \\
0 & 0 & 0 & I & 0 & 0 & 0 & A_{3}(5,6,7, \ldots) \\
0 & 2 E_{1,3} & 0 & 0 & B_{2}(5,6,8) & B_{1}(5,6,7, \ldots) & B_{2}(7,9,10, \ldots) & B_{3}(5,6,7, \ldots)
\end{array}\right) .
\end{aligned}
$$

Here $E_{i, j}$ is the matrix having a one in place $(i, j)$ and zeros everywhere else. Further,

$$
A_{3}(k, l, m, \ldots)=\left(\begin{array}{cccc}
\widetilde{I}_{k}^{1} & \widetilde{I}_{l}^{1} & \widetilde{I}_{m}^{1} & \ldots \\
\widetilde{I}_{k}^{2} & \widetilde{I}_{l}^{2} & \widetilde{I}_{m}^{2} & \ldots \\
\widetilde{I}_{k}^{3} & \widetilde{I}_{l}^{3} & \widetilde{I}_{m}^{3} & \ldots
\end{array}\right),
$$

while the rows of $B_{j}(k, l, \ldots)$ are given by the invariantization of

$$
\left(\widetilde{D}_{k}\left(v^{\alpha} v^{\beta}\right) \quad \widetilde{D}_{l}\left(v^{\alpha} v^{\beta}\right) \quad \ldots\right), \quad \alpha, \beta=1,2,3
$$


after all the differentiations have been performed.

The next step is to row reduce $W$ so that the initial $15 \times 15$ minor equals the identity. Then $P=(I, \widehat{P})$, where $\widehat{P}$ is the invariantization of the matrix

$$
\widehat{H}=\left(\begin{array}{ccc}
0 & 0 & 0 \\
H_{11} & 0 & 0 \\
H_{21} & H_{22} & H_{23} \\
0 & 0 & H_{33} \\
H_{41} & H_{42} & H_{43}
\end{array}\right) .
$$

The columns of $\widehat{H}$ correspond to $\tilde{u}_{k}^{1}, k=5,6,7, \ldots, \tilde{u}_{k}^{2}, k=7,9,10, \ldots$ and $\tilde{u}_{k}^{3}, k=5,6,7, \ldots$ in precisely that order. Explicitly,

$$
H_{11}=H_{33}=\left(\begin{array}{cccccc}
\tilde{v}_{5}^{1} & \tilde{v}_{6}^{1} & \tilde{v}_{7}^{1} & \tilde{v}_{8}^{1} & \tilde{v}_{9}^{1} & \cdots \\
0 & 0 & \tilde{v}_{7}^{2} & 0 & \tilde{v}_{9}^{2} & \ldots \\
\tilde{v}_{5}^{3} & \tilde{v}_{6}^{3} & \tilde{v}_{7}^{3} & \tilde{v}_{8}^{3} & \tilde{v}_{9}^{3} & \ldots
\end{array}\right)
$$

Further, $H_{21}$ and $H_{41}$ have as columns

$$
\left(\begin{array}{c}
-\tilde{v}_{5}^{1}\left[\widetilde{D}_{k}\left(v^{1}\right)^{2}-2 \tilde{v}_{k}^{3}\right]-\frac{1}{2} \tilde{v}_{6}^{1} \widetilde{D}_{k}\left(v^{1} v^{2}\right)-\tilde{v}_{8}^{1} \widetilde{D}_{k}\left(v^{1} v^{3}\right) \\
0 \\
-\tilde{v}_{5}^{3}\left[\widetilde{D}_{k}\left(v^{1}\right)^{2}-2 \tilde{v}_{k}^{3}\right]-\frac{1}{2} \tilde{v}_{6}^{3} \widetilde{D}_{k}\left(v^{1} v^{2}\right)-\tilde{v}_{8}^{3} \widetilde{D}_{k}\left(v^{1} v^{3}\right)
\end{array}\right) \quad\left(\begin{array}{c}
\widetilde{D}_{k}\left(v^{1}\right)^{2}-2 \tilde{v}_{k}^{3} \\
\frac{1}{2} \widetilde{D}_{k}\left(v^{1} v^{2}\right) \\
\widetilde{D}_{k}\left(v^{1} v^{3}\right)
\end{array}\right)
$$

respectively, for $k=5,6, \ldots ; H_{22}$ and $H_{42}$ have columns equal to

$$
\left(\begin{array}{c}
\tilde{v}_{k}^{1}-\tilde{v}_{5}^{1} \widetilde{D}_{k}\left(v^{1} v^{2}\right)-\frac{1}{2} \tilde{v}_{6}^{1} \widetilde{D}_{k}\left(v^{2}\right)^{2}-\tilde{v}_{8}^{1} \widetilde{D}_{k}\left(v^{2} v^{3}\right) \\
\tilde{v}_{k}^{2} \\
\tilde{v}_{k}^{3}-\tilde{v}_{5}^{3} \widetilde{D}_{k}\left(v^{1} v^{2}\right)-\frac{1}{2} \tilde{v}_{6}^{3} \widetilde{D}_{k}\left(v^{2}\right)^{2}-\tilde{v}_{8}^{3} \widetilde{D}_{k}\left(v^{2} v^{3}\right)
\end{array}\right) \quad\left(\begin{array}{c}
\widetilde{D}_{k}\left(v^{1} v^{2}\right) \\
\frac{1}{2} \widetilde{D}_{k}\left(v^{2}\right)^{2} \\
\widetilde{D}_{k}\left(v^{2} v^{3}\right)
\end{array}\right)
$$

respectively, for $k=7,9,10, \ldots$ Finally $H_{23}$ and $H_{43}$ have, respectively, columns given by

$$
\left(\begin{array}{c}
-\tilde{v}_{5}^{1} \widetilde{D}_{k}\left(v^{1} v^{3}\right)-\frac{1}{2} \tilde{v}_{6}^{1} \widetilde{D}_{k}\left(v^{2} v^{3}\right)-\tilde{v}_{8}^{1} \widetilde{D}_{k}\left(v^{3}\right)^{2} \\
0 \\
-\tilde{v}_{5}^{3} \widetilde{D}_{k}\left(v^{1} v^{3}\right)-\frac{1}{2} \tilde{v}_{6}^{3} \widetilde{D}_{k}\left(v^{2} v^{3}\right)-\tilde{v}_{8}^{3} \widetilde{D}_{k}\left(v^{3}\right)^{2}
\end{array}\right) \quad\left(\begin{array}{c}
\widetilde{D}_{k}\left(v^{1} v^{3}\right) \\
\frac{1}{2} \widetilde{D}_{k}\left(v^{2} v^{3}\right) \\
\widetilde{D}_{k}\left(v^{3}\right)^{2}
\end{array}\right)
$$

for $k=5,6,7, \ldots$

The function $M_{K ; x}^{\alpha}$ equals minus the dot product of the first row of $T$ and the column in $P$ corresponding to $u_{K}^{\alpha}$. $M_{K ; y}^{\alpha}$ would be found similarly using the second row of $T$. The most interesting of the resulting recurrence 
Differential invariants for parametrized projective surfaces

formulae are the following ones:

$$
\begin{aligned}
D_{x} I_{x y}^{1} & =I_{x x y}^{1}-I_{x y}^{3} I_{x x x}^{1}-2\left(I_{x y}^{3}\right)^{2} I_{x x x}^{2}+\frac{1}{2} I_{y y}^{3} I_{x x x}^{2}-\frac{1}{2} I_{x y y}^{2} \\
D_{y} I_{x y}^{1} & =I_{x y y}^{1}-\left(I_{x y}^{1}\right)^{2}-I_{x y}^{3} I_{x x y}^{1}+2 I_{x y}^{3} I_{x y y}^{2}-\frac{1}{2} I_{y y y}^{2} \\
D_{x} I_{x y}^{3} & =I_{x x y}^{3}-I_{x y}^{1}-I_{x y}^{3} I_{x x x}^{3} \\
D_{y} I_{x y}^{3} & =I_{x y y}^{3}-\left(I_{x y}^{1} I_{x y}^{3}\right)-I_{x y}^{3} I_{x x y}^{3} \\
D_{x} I_{y y}^{1} & =I_{x y y}^{1}-\left(I_{y y}^{3} I_{x x x}^{1}\right)-2 I_{x y}^{3} I_{y y}^{3} I_{x x x}^{2} \\
D_{y} I_{y y}^{1} & =I_{y y y}^{1}-\left(I_{x y}^{1} I_{y y}^{1}\right)-I_{y y}^{3} I_{x x y}^{1}+2 I_{y y}^{3} I_{x y y}^{2} \\
D_{x} I_{y y}^{3} & =I_{x y y}^{3}-I_{y y}^{1}-I_{y y}^{3} I_{x x x}^{3} \\
D_{y} I_{y y}^{3} & =I_{y y y}^{3}-\left(I_{y y}^{1} I_{x y}^{3}\right)-I_{y y}^{3} I_{x x y}^{3} \\
D_{x} I_{x x x}^{2} & =I_{x x x x}^{2}-I_{x x x}^{2} I_{x x x}^{3} \\
D_{y} I_{x x x}^{2} & =I_{x x x y}^{2} \\
D_{x} I_{x y y}^{2} & =I_{x x y y}^{2}+2 I_{x y}^{1} I_{x y}^{3} I_{x x x}^{2}+2 I_{x y}^{3} I_{x x x}^{2} I_{x x y}^{3}-I_{x x x}^{2} I_{x y y}^{3}-2 I_{x y}^{3} I_{x x x y}^{2} \\
D_{y} I_{x y y}^{2} & =I_{x y y y}^{2}-2 I_{x y}^{1} I_{x y y}^{2}-2 I_{x y}^{3} I_{x x y y}^{2} \\
D_{x} I_{y y y}^{2} & =I_{x y y y}^{2}+3 I_{y y}^{1} I_{x y}^{3} I_{x x x}^{2}+3 I_{y y}^{3} I_{x x x}^{2} I_{x x y}^{3}-I_{x x x}^{2} I_{y y y}^{3}-3 I_{y y}^{3} I_{x x x y}^{2} \\
D_{y} I_{y y y}^{2} & =I_{y y y y}^{2}-3 I_{y y}^{1} I_{x y y}^{2}-3 I_{y y}^{3} I_{x x y y}^{2}
\end{aligned}
$$

These imply that we can reduce our generating set to be

$$
I_{x y}^{1}, \quad I_{y y}^{1}, \quad I_{x y}^{3}, \quad I_{y y}^{3}, \quad I_{x x x}^{2}, \quad I_{x y y}^{2}, \quad I_{y y y}^{2}
$$

Finally, if one wishes to find $M_{K ; x x}^{i}$ the process will have to be iterated. For example,

$$
D_{x} I_{K, x}^{\alpha}=I_{K,\{x x\}}^{\alpha}+M_{\{K ; x\} ; x}^{\alpha}+D_{x} M_{K ; x}^{\alpha}
$$

so that

$$
M_{K ;\{x x\}}^{\alpha}=M_{\{K ; x\} ; x}^{\alpha}+D_{x} M_{K ; x}^{\alpha} .
$$

Now, as for the complete classification of syzygies, only the second case in Theorem 3.6 applies here, since all phantom invariants have lower or equal order than the generating invariants. There are a wide variety of syzygies, but they can all be written in terms of differential consequences of the ones 
involving the generating set (6.14); namely

$$
\begin{aligned}
D_{y} I_{x y}^{\alpha}-D_{x} I_{y y}^{\alpha} & =M_{x y ; y}^{\alpha}-M_{y y ; x}^{\alpha}, \quad \alpha=1,3 \\
D_{y} I_{x y y}^{2}-D_{x} I_{y y y}^{2} & =M_{x y y ; y}^{2}-M_{y y y ; x}^{2} \\
D_{y}^{2} I_{x x x}^{2}-D_{x}^{2} I_{x y y}^{2} & =M_{x x x ; y y}^{2}-M_{x y y ; x x}^{2} \\
D_{y}^{3} I_{x x x}^{2}-D_{x}^{3} I_{y y y}^{2} & =M_{x x x ; y y y}^{2}-M_{y y y ; x x x}^{2} .
\end{aligned}
$$

\section{Surfaces in $\mathbb{R} \mathbb{P}^{n-1}$.}

Now let us treat the general case of parametrized projective surfaces in $\mathbb{R} P^{n-1}$. We retain the order on derivatives and notation introduced in Section 5 . We first extend the result in Section 6 for the case $n=4$.

Definition 7.1. A surface $u(x, y)$ is nondegenerate if the generalized Wronskian matrix

$$
U_{n-1}=\left(\begin{array}{cccc}
\tilde{u}_{2}^{1} & \tilde{u}_{3}^{1} & \ldots & \tilde{u}_{n}^{1} \\
\tilde{u}_{2}^{2} & \tilde{u}_{3}^{2} & \ldots & \tilde{u}_{n}^{2} \\
\vdots & \vdots & \ddots & \vdots \\
\tilde{u}_{2}^{n-1} & \tilde{u}_{3}^{n-1} & \ldots & \tilde{u}_{n}^{n-1}
\end{array}\right)
$$

is nonsingular:

$$
\Delta_{n-1}=\operatorname{det} U_{n-1} \neq 0
$$

Theorem 7.2. The projective group action on $\mathbb{R} \mathbb{P}^{n-1}$ stabilizes at order $m+1$, where $m=\mu(n)$. Therefore an equivariant moving frame $\rho^{(k)}: \mathrm{J}^{k} \rightarrow$ $\mathbb{R P}^{n-1}$ will exist only for $k \geq m+1$.

Proof. The proof is identical to that in the case $n=4$. One must show that the lowest order for which the prolongations of the infinitesimal generators (5.8) are independent is $m+1$. Under the condition $\Delta_{n-1} \neq 0$ it is not hard to see that $\mathrm{pr}^{(k)} \mathbf{v}_{\alpha}$ and $\mathrm{pr}^{(k)} \mathbf{v}_{\alpha \beta}$ are all independent (one would write the matrix of coefficients and would check that it has maximal rank). On the other hand, if $f_{1}, \ldots, f_{n-1}$ are functions satisfying $\sum_{i=1}^{n-1} f_{i} u_{k}^{i}=0$, for any $k \leq m$, then it is not hard to show that $\sum_{i=1}^{n-1} f_{i} \operatorname{pr}^{(k)} \mathbf{w}_{\alpha}$ is a combination of the $\operatorname{pr}^{(k)} \mathbf{v}_{\alpha}$ and $\operatorname{pr}^{(k)} \mathbf{v}_{\alpha \beta}$. If $k=m+1$ this will not happen and one can prove that the rank of the coefficient matrix is maximal. 
As in the previous section, we want to normalize some of the derivatives of the lifted invariants (5.6), and fix, through these normalizations the values of the parameters $a_{j}^{i}$. Once we have done that, the invariants will be given by the substitution of these parameters in the remaining lifted invariants. We write the matrix $A$ in block form

$$
A=\left(\begin{array}{cc}
\widetilde{A} & \mathbf{a} \\
\widetilde{\mathbf{a}} & a_{n}^{n}
\end{array}\right)
$$

where $\widetilde{A}$ denotes the upper $(n-1) \times(n-1)$ minor. First of all, normalizing the zero ${ }^{\text {th }}$ order lifted invariants $v=0$ will specify the column vector $\mathbf{a}$. Then we will require $\tilde{v}_{k}^{\alpha}=\delta_{k}^{\alpha}, \alpha=1,2, \ldots, n-1, k=2,3, \ldots, n$, which will normalize the minor $\widetilde{A}$. Finally, we will give $n-1$ higher order normalizations that will allow us to find values for the bottom row vector $\widetilde{\mathbf{a}}$. The last entry, $a_{n}^{n}$, will be fixed so that the determinant of $A$, after normalization, remains equals 1.

In view of (5.6), the normalizations $v=0$ are achieved by setting

$$
a_{n}^{\alpha}=-\sum_{\beta=1}^{n-1} a_{\beta}^{\alpha} u^{\beta}, \quad \alpha=1, \ldots, n-1 .
$$

As before, let us call

$$
K_{1}=K=\sum_{\beta=1}^{n-1} a_{\beta}^{n-1} u^{\beta}+a_{n-1}^{n-1}, \quad K_{k}=\widetilde{D}_{k} K=\sum_{\beta=1}^{n-1} a_{\beta}^{n-1} u_{k}^{\beta} .
$$

Notice that $\widetilde{D}_{1}$ is the $(0,0)$ differentiation, following the lexicographical order. The value of $K$ will be fixed at the end of the process.

Next, we will impose the normalization

$$
V_{n-1}=\left(\begin{array}{cccc}
\tilde{v}_{2}^{1} & \tilde{v}_{3}^{1} & \ldots & \tilde{v}_{n}^{1} \\
\tilde{v}_{2}^{2} & \tilde{v}_{3}^{2} & \ldots & \tilde{v}_{n}^{2} \\
\vdots & \vdots & \ddots & \vdots \\
\tilde{v}_{2}^{n-1} & \tilde{v}_{3}^{n-1} & \ldots & \tilde{v}_{n}^{n-1}
\end{array}\right)=I .
$$

The result of the first order normalizations can be stated using the notation introduced in (5.4), (5.5) above.

Theorem 7.3. Assume that (7.4) and (7.2) hold. Then,

$$
\widetilde{A}=\mathbf{K} \cdot\left(U_{n-1}\right)^{-1},
$$


where $\mathbf{K}$ is the matrix whose $(k, l)$ entry equals $\llbracket\left[\begin{array}{c}k \\ l\end{array}\right] K_{\tau}(k, l), k, l=$ $1,2, \ldots, n-1$. In particular, the entry is zero if $\llbracket\left[\begin{array}{c}k \\ l\end{array} \rrbracket=0\right.$, or, equivalently, $\tau(k, l)$ is not defined.

Proof. Applying Leibniz' rule to

$$
\widetilde{D}_{k}\left(K v^{\alpha}\right)=\sum_{\beta=1}^{n-1} a_{\beta}^{\alpha} \tilde{u}_{k}^{\beta}
$$

produces the following formula:

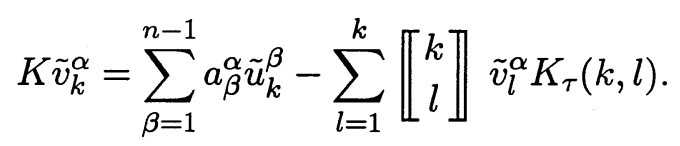

Therefore, applying the normalizations (7.4), (7.2), we obtain

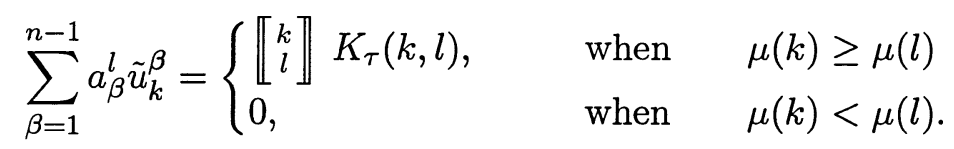

Thus, the analogy of equation (6.8) in the general case is

$$
\widetilde{A} \cdot U_{n-1}=\left(\begin{array}{cccc}
a_{1}^{1} & a_{2}^{1} & \ldots & a_{n-1}^{1} \\
a_{1}^{2} & a_{2}^{2} & \ldots & a_{n-1}^{2} \\
\vdots & \vdots & \ddots & \vdots \\
a_{1}^{n-1} & a_{2}^{n-1} & \ldots & a_{n-1}^{n-1}
\end{array}\right)\left(\begin{array}{cccc}
\tilde{u}_{2}^{1} & \tilde{u}_{3}^{1} & \ldots & \tilde{u}_{n}^{1} \\
\tilde{u}_{2}^{2} & \tilde{u}_{3}^{2} & \ldots & \tilde{u}_{n}^{2} \\
\vdots & \vdots & \ddots & \vdots \\
\tilde{u}_{2}^{n-1} & \tilde{u}_{3}^{n-1} & \ldots & \tilde{u}_{n}^{n-1}
\end{array}\right)=\mathbf{K}
$$

where $\mathbf{K}$ is given as in the statement of the Theorem.

In order to understand the last group of normalizations, it will help us to discuss the form of the $(n-1) \times(n-1)$ matrix $\mathbf{K}$ in some detail. Let us represent its nonzero entries by a $*$. Recall from the notations that the triangular number $t_{k}$ represents the number of derivatives of order less than $k$. That is, $t_{k}+1, t_{k}+2, \ldots, t_{k+1}$ are the positions in the lexicographical order for derivatives of order $k$. Using this ordering of derivatives and conditions 
for when $\left.\left[\begin{array}{l}k \\ l\end{array}\right]\right]=0$, we readily see that $\mathbf{K}$ has the shape

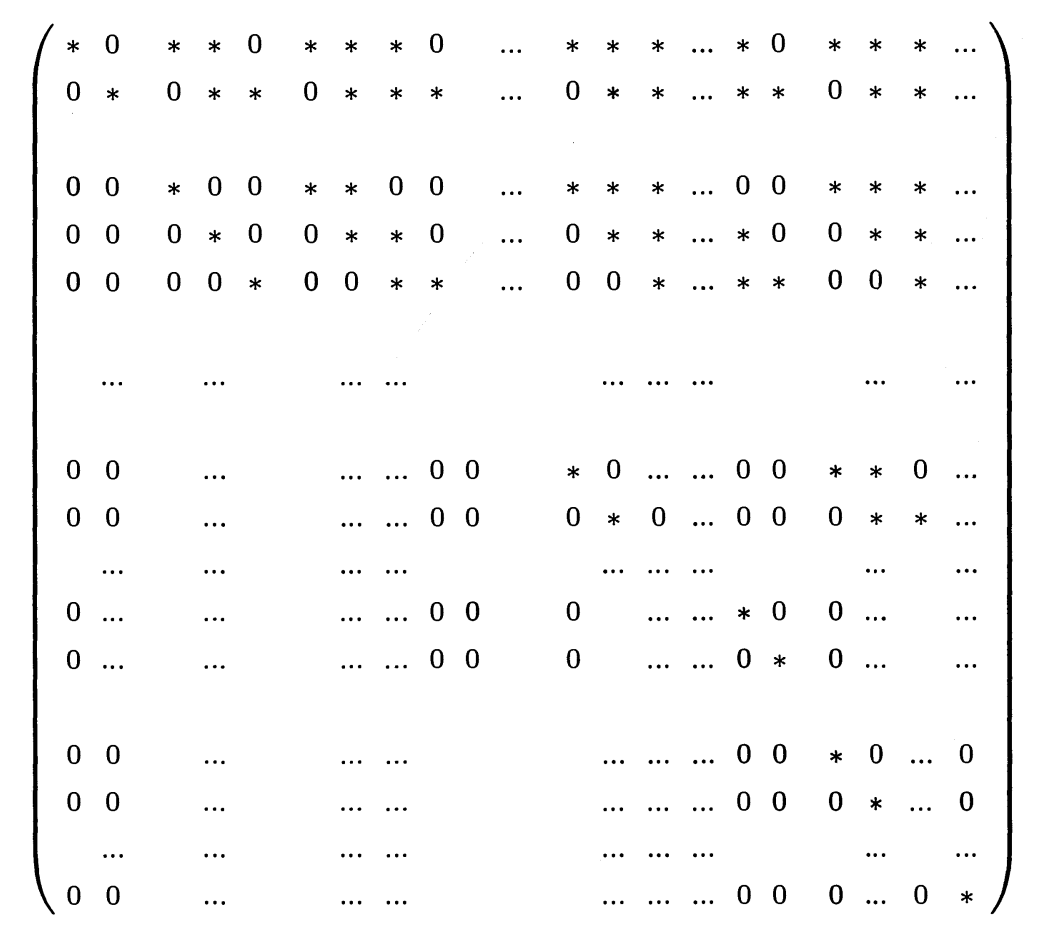

where the block in (block) place $(i, j)$ is of size $(i+1) \times(j+1)$ and where, if $\mu(n)=m$, there are $m$ by $m$ blocks.

In particular, $\mathbf{K}$ is upper triangular, and the diagonal entries all equal $K$. Therefore $\operatorname{det} \mathbf{K}=K^{n-1}$, and hence, in view of our order zero normalization (7.5), $A \in \mathrm{SL}(n)$ if and only if

$$
K=\left(\Delta_{n-1}\right)^{-1 / n}
$$

Thus,

$$
a_{n}^{n}=K-\sum_{i=1}^{n-1} a_{i}^{n} u^{i} .
$$

Moreover, the derivatives $K_{1}, K_{2}, K_{3}, \ldots$, multiplied by appropriate binomial coefficients, appear one after another in order, in each row, in the indicated nonzero slots. This way, we can determine how many different $K_{j}^{\prime} s$ appear in the normalization of each one of the parameters in $\widetilde{A}$ by simply counting how many nonzero entries there are in the appropriate row of $\mathbf{K}$. Notice that, for example, if $\mu(n)=m$, and if $n-t_{m}<m$, the coefficients $a_{i}^{t_{m}-1}$ will involve only $K$, if $n-t_{m}=m$, these coefficients will involve $K$ 
and $K_{x}$, and if $n-t_{m}=m+1$ (that is $n=t_{m+1}$ ), the coefficients will involve $K, K_{x}$ and $K_{y}$, and these would be the only possible cases for $a_{i}^{t_{m}-1}$. The same way, by simply counting how many nonzero (or zero) entries there are in the $k$ th row, we can know how many different $K_{j}$ 's, and which ones, are used in the definition of the parameters $a_{i}^{k}$.

Lemma 7.4. Suppose $t_{m}<n \leq t_{m+1}$. Then

$$
n-t_{s}-s(m-s+1) \leq t_{m-s+1}
$$

for all $s=1,2, \ldots, m-1$.

Proof. The proof is a simple calculation. Observe that $n-t_{m} \leq m+1$, since $m+1$ is the difference between $t_{m}$ and $t_{m+1}$. Also, we have that

$$
\begin{aligned}
n-t_{s} & =n-t_{m}+\left(t_{m}-t_{m-1}\right)+\left(t_{m-1}-t_{m-2}\right)+\cdots+\left(t_{s+1}-t_{s}\right) \\
& =n-t_{m}+m+(m-1)+\cdots+(s+1) \\
& =n-t_{m}+\frac{(m+s+1)(m-s)}{2} .
\end{aligned}
$$

Therefore

$$
\begin{aligned}
n-t_{s}-s(m-s+1) & =n-t_{m}+\frac{(m+s+1)(m-s)}{2}-s(m-s)-s \\
& \leq m+1+\frac{(m-s)(m-s+1)}{2}-s=t_{m-s+1} .
\end{aligned}
$$

This suffices to prove the result.

To make the description of the frame more accessible to the reader we will separate the normalizations in three different groups.

Case 1.

Assume that $n=t_{m+1}$. Consider the normalizations

$$
\begin{aligned}
& v_{J}^{t_{s+1}-1}=0 \\
& J=(m-s+2, s-1),(m-s+1, s), \ldots,(1, m), \quad s=1, \ldots, m .
\end{aligned}
$$

That is, we normalize the derivatives $\tilde{v}_{r}^{t_{s+1}-1}$ for $r=t_{m+1}+s, t_{m+1}+s+$ $1, \ldots, t_{m+1}+m+1$. 
Theorem 7.5. The normalizations (7.8) determine $K_{j}=k_{j}\left(u^{(m+1)}\right)$ as functions of $u$ and its derivatives up to order $m+1$, for all $j=2, \ldots, n$. Indeed,

$$
\left(\begin{array}{llll}
a_{1}^{n} & a_{2}^{n} & \ldots & a_{n-1}^{n}
\end{array}\right)=\mathbf{k} U_{n-1}^{-1},
$$

where $\mathbf{k}=\left(k_{j}\right)$. These values of the parameters, together with

$$
a_{n}^{n}=\Delta_{n-1}^{-1 / n}-\Delta_{n-1}^{-1} \mathbf{k} \cdot \Delta_{0}
$$

where $\Delta_{\mathbf{0}}=\left(\begin{array}{c}\Delta_{n-1}(2,1) \\ \Delta_{n-1}(3,1) \\ \vdots \\ \Delta_{n-1}(n, 1)\end{array}\right)$, and where $\Delta_{n-1}(j, 1)$ is the determinant ob-

tained from $\Delta_{n-1}$ by substituting the column with the $j^{\text {th }}$ derivative by the column without differentiation, define an equivariant frame of order $m+1$.

Proof. Formula 7.7 implies that such a normalization will enable us to write $K_{\tau(r, k)}$ in terms of as many of the $K_{j}$ as will appear in the formula for the coefficients $a_{1}^{k}, \ldots, a_{n-1}^{k}$. If $k=t_{s+1}-1$, then these coefficients are written in terms of $K_{1}, \ldots, K_{n-t_{s}-s(m-s+1)}$, since there are exactly $n-t_{s}-s(m-$ $s+1)$ nonzero entries in each $t_{s+1}-1$ row. Lemma 7.4 and (7.7) imply that normalizations (7.8) will express $K_{t_{m-s+1}+1}, \ldots, K_{t_{m-s+2}}$ in terms of previous $K$ 's, namely, $K_{1}, \ldots K_{t_{m-s+1}}$.

In this manner, the condition

$$
\tilde{v}_{t_{m+1}+m}^{t_{m+1}-1}=\tilde{v}_{t_{m+1}+m+1}^{t_{m+1}-1}=0
$$

(case $s=m$ ) produces $K_{2}$ and $K_{3}$ in terms of $a_{i}^{n-1}$ and the derivatives $\tilde{u}_{t_{m+1}+m}^{i}, \tilde{u}_{t_{m+1}+m+1}^{i}, i=1,2, \ldots, n-1$. But the formulae for $a_{i}^{n-1}$ in Theorem 7.3, and that of $K_{1}$, imply that

$$
a_{i}^{n-1}=\Delta_{n-1}^{-(n+1) / n} C_{i, n-1}\left(U_{n-1}\right)
$$

so that

$$
\begin{aligned}
& K_{2}=k_{2}=\frac{1}{2} \Delta_{n-1}^{-(n+1) / n} \Delta_{n-1}(n-1, n+m) \\
& K_{3}=k_{3}=\frac{1}{m} \Delta_{n-1}^{-(n+1) / n} \Delta_{n-1}(n-1, n+m+1)
\end{aligned}
$$


where $\Delta_{n-1}(i, j)$ is the determinant of the matrix obtained from $U$ when we substitute the column with $i$ derivatives by the column with $j$ derivatives, and $C_{i, j}(U)$ is the cofactor of the matrix $U$ associated to the $(i, j)$ entry.

Next, the normalizations

$$
\tilde{v}_{t_{m+1}+m-1}^{t_{m}-1}=\tilde{v}_{t_{m+1}+m}^{t_{m}-1}=\tilde{v}_{t_{m+1}+m+1}^{t_{m}-1}=0
$$

will produce $K_{4}, K_{5}$ and $K_{6}$ in terms of $K_{2}$ and $K_{3}$, namely

$$
\begin{aligned}
& \sum_{i=1}^{n-1} a_{i}^{t_{m}-1} \tilde{u}_{t_{m+1}+m-1}^{i}=3 K_{4}, \\
& \sum_{i=1}^{n-1} a_{i}^{t_{m}-1} \tilde{u}_{t_{m+1}+m}^{i}=2(m-1) K_{5}, \\
& \sum_{i=1}^{n-1} a_{i}^{t_{m}-1} \tilde{u}_{t_{m+1}+m+1}^{i}=\left(\begin{array}{c}
m \\
m-2
\end{array}\right) K_{6} .
\end{aligned}
$$

The value of $a_{i}^{t_{m}-1}$ has been already fixed to be

$$
\begin{aligned}
a_{i}^{t_{m}-1}= & \Delta_{n-1}^{-(n+1) / n} C_{i, t_{m}-1}\left(U_{n-1}\right) \\
& +\Delta_{n-1}^{-1}\left[2 K_{2} C_{i, n-2}\left(U_{n-1}\right)+(m-1) K_{3} C_{i, n-1}\left(U_{n-1}\right)\right]
\end{aligned}
$$

and therefore

$$
\begin{aligned}
k_{4}= & \frac{1}{3} \Delta_{n-1}^{-(n+1) / n} \Delta_{n-1}\left(t_{m}-1, n+m-1\right) \\
& +\frac{1}{3} \Delta_{n-1}^{-1}\left[2 k_{2} \Delta_{n-1}(n-2, n+m-1)\right. \\
& \left.\quad+(m-1) k_{3} \Delta_{n-1}(n-1, n+m-1)\right], \\
k_{5}= & \frac{1}{2} \Delta_{n-1}^{-(n+1) / n} \Delta_{n-1}\left(t_{m}-1, n+m\right) \\
& +\Delta_{n-1}^{-1}\left[\frac{1}{m-1} k_{2} \Delta_{n-1}(n-2, n+m)+\frac{1}{2} k_{3} \Delta_{n-1}(n-1, n+m)\right], \\
k_{6}= & \frac{2}{m(m-1)} \Delta_{n-1}^{-(n+1) / n} \Delta_{n-1}\left(t_{m}-1, n+m+1\right)+ \\
& +\frac{2}{m(m-1)} \Delta_{n-1}^{-1}\left[2 k_{2} \Delta_{n-1}(n-2, n+m+1)\right. \\
& \left.\quad+(m-1) k_{3} \Delta_{n-1}(n-1, n+m+1)\right] .
\end{aligned}
$$


They can be expressed in terms of derivatives of $u$ up to order $m+1$, upon substitution of $k_{2}$ and $k_{3}$.

And so the substitution proceeds. At each step we solve for some of the $K_{j}$ in terms of the previous ones, until we reach the case $s=1$, in which we solve for $K_{r}, r=t_{m}+1, \ldots, t_{m+1}=n$, in terms of $K_{1}, \ldots, K_{t_{m}}$. Since the entries of $\mathbf{k}$ are generated by this recursion, they can be explicitly obtained upon inversion of a lower triangular matrix, whose entries are written in terms of determinants of the form $\Delta_{n-1}(i, j)$. Indeed, if we assume we have normalized up to $\tilde{v}_{r}^{t_{s+1}-1}=0, r=n+s, n+s+1, \ldots, n+m+1$, obtaining the values of $k_{t_{m-s+1}+1}, \ldots, k_{t_{m-s+2}}$ in terms of $k_{2}, \ldots, k_{t_{m-s+1}}$, then the normalizations

$$
\tilde{v}_{r}^{t_{s}-1}=0, \quad r=n+s-1, n+s, \ldots, n+m+1,
$$

will produce the relationship

$$
\sum_{i=1}^{n-1} a_{i}^{t_{s}-1} \tilde{u}_{t_{m+1}+s+k-2}^{i}=\llbracket\left[\begin{array}{c}
t_{m+1}+s+k-2 \\
t_{m-s+2}+k
\end{array} \rrbracket K_{t_{m-s+2}+k}\right.
$$

for all $k=1,2, \ldots, m-s+3$. On the other hand we have that $a_{i}^{t_{s}-1}$ equals

$$
\begin{aligned}
\Delta_{n-1}^{-1}( & \Delta_{n-1}^{-\frac{1}{n}} C_{i, t_{s}-1}\left(U_{n-1}\right) \\
& \quad+\sum_{r=1}^{m-s+1} \sum_{l=1}^{r+1} \llbracket\left[\begin{array}{c}
t_{r+s-1}+s+l-2 \\
t_{s}-1
\end{array} \rrbracket k_{t_{r}+l} C_{i, t_{r+s-1}+s+l-2}\left(U_{n-1}\right)\right) .
\end{aligned}
$$

Hence, we obtain the recursion formula for the coefficients of $\mathbf{k}$, namely

$$
\begin{aligned}
& k_{t_{m-s+2}+k}=(m-s-k+4)^{-1}\left(\begin{array}{c}
s+k-3 \\
k-1
\end{array}\right)^{-1} \Delta_{n-1}^{-1} \\
& \cdot\left[\Delta_{n-1}^{-\frac{1}{n}} \Delta_{n-1}\left(t_{s}-1, n+s+k-2\right)+\sum_{r=1}^{m-s+1} \sum_{l=1}^{r+1}(r-l+2)\left(\begin{array}{c}
s+l-3 \\
s-2
\end{array}\right)\right. \\
& \left.\cdot k_{t_{r}+l} \Delta_{n-1}\left(t_{r+s-1}+s+l-2, n+s+k-2\right)\right]
\end{aligned}
$$

for all $k=1,2, \ldots, m-s+3$.

This formula can be easily written in a matrix formulation of the form $\mathbf{k}=\mathbf{b}+B \mathbf{k}$, where $\mathbf{k}$ and $\mathbf{b}$ are vectors, the latter being defined by the first 
term in the equation (7.11), and where $B$ is a strictly lower triangular matrix. whose entries are written in terms of $\Delta_{n-1}(i, j)$. Hence $\mathbf{k}=(I-B)^{-1} \mathbf{b}$ can be explicitly obtained. At the end we have produced a vector $\mathbf{k}=$ $\left(k_{j}\right)$ defining the values of $K_{2}, K_{3}, \ldots, K_{n}$ appearing in our frame when we substitute them in the matrix $\mathbf{K}$. This vector will also determine the values of the parameters $a_{1}^{n}, \ldots, a_{n}^{n}$. Namely

$$
\left(\begin{array}{llll}
a_{1}^{n} & a_{2}^{n} & \ldots & a_{n}^{n}
\end{array}\right) A_{n-1}=\mathbf{k}, \quad\left(\begin{array}{llll}
a_{1}^{n} & a_{2}^{n} & \ldots & a_{n}^{n}
\end{array}\right)=\mathbf{k} A_{n-1}^{-1},
$$

and

$$
a_{n}^{n}=K-\sum_{i=1}^{n-1} a_{i}^{n} u^{i}=\Delta_{n-1}^{-\frac{1}{n}}-\Delta_{n-1}^{-1} \mathbf{k}\left(\begin{array}{c}
\Delta_{n-1}(2,1) \\
\Delta_{n-1}(3,1) \\
\vdots \\
\Delta_{n-1}(n, 1)
\end{array}\right) .
$$

This completes the proof of case 1.

Case 2.

Assume that $n=t_{m}+m$. In this case we simply have to repeat the procedure above, once normalizations (7.8) have been substituted by the normalizations

$$
\begin{aligned}
& \tilde{v}_{t_{m+1}+1}^{t_{m}+1}=0=\tilde{v}_{t_{m+1}}^{t_{m}} \tilde{v}_{J}^{t_{s+1}-1}=0 \\
& J=(m-s+2, s-1),(m-s+1, s), \ldots,(2, m-1),(1, m) \\
& \quad s=2, \ldots, m-1
\end{aligned}
$$

and

$$
\tilde{v}_{J}^{t_{2}-1}=0, \quad J=(m+1,0),(m, 1), \ldots,(2, m-1) .
$$

Case 3.

Assume that $n=t_{m}+k$ for some $k=1,2, \ldots, m-1$. Again, repeat the procedure followed in the first case, but now the normalizations are somehow more involved. They are given by

$$
\begin{aligned}
& \tilde{v}_{J}^{t_{s+1}-1}=0 \\
& J=(m-k+1, k-1),(m-k, k), \ldots,(1, m-1), \\
& \quad(m-s+2, s-1),(m-s+1, s), \ldots,(m-k+2, k-1)
\end{aligned}
$$

whenever $s=1,2, \ldots, k$, and

$$
\tilde{v}_{J}^{t_{s+1}-1}=0, \quad J=(m-s+1, s-1),(m-s, s), \ldots,(1, m-1),
$$


whenever $s=k+1, \ldots, m-1$. This gives us a total of $t_{m}+k-1$ normalizations that, as we indicated in the first case, will generate values for $K_{j}$, $j=2, \ldots, n$ in terms of $u^{(m+1)}$ and, hence, will determine the rest of the parameters involved in the construction of the frame.

\section{Conclusions.}

In this paper we have described the regularized moving frame method. Using this method, we have computed complete systems of generating differential invariants and invariant differential operators for the geometry of two-dimensional surfaces in projective space. These results can be directly applied to problems of equivalence, symmetry and rigidity of projective surfaces.

As outlined in the introduction, there are important implications of our result in the study of generalizations of AGD evolutions to the case of two independent variables. For example, in the case $n=3$, one can obtain the most general formula for invariant evolutions for maps $u: \mathbb{R}^{2} \rightarrow \mathbb{R} \mathbb{P}^{2}$. For that we use the same approach used in [12] and deduce that the most general invariant evolution is given by

$$
\left(\begin{array}{l}
u^{1} \\
u^{2}
\end{array}\right)_{t}=\left(\begin{array}{ll}
u_{x}^{1} & u_{y}^{1} \\
u_{x}^{2} & u_{y}^{2}
\end{array}\right)\left(\begin{array}{l}
J_{1} \\
J_{2}
\end{array}\right)
$$

where $J_{1}$ and $J_{2}$ are two general differential invariants for the action. From our results in this paper, we can conclude that a generating set of differential invariants is given by the four independent functionals

$$
\begin{aligned}
& I_{1}=\frac{\left|\begin{array}{ll}
u_{x}^{1} & u_{x x}^{1} \\
u_{x}^{2} & u_{x x}^{2}
\end{array}\right|}{\left|\begin{array}{ll}
u_{x}^{1} & u_{y}^{1} \\
u_{x}^{2} & u_{y}^{2}
\end{array}\right|}, \quad I_{3}=\frac{\left|\begin{array}{cc}
u_{x y}^{1} & u_{y}^{1} \\
u_{x y}^{2} & u_{y}^{2}
\end{array}\right|-\frac{1}{2}\left|\begin{array}{cc}
u_{x}^{1} & u_{y y}^{1} \\
u_{x}^{2} & u_{y y}^{2}
\end{array}\right|}{\left|\begin{array}{ll}
u_{x}^{1} & u_{y}^{1} \\
u_{x}^{2} & u_{y}^{2}
\end{array}\right|} \\
& I_{2}=\frac{\left|\begin{array}{ll}
u_{y}^{1} & u_{y y}^{1} \\
u_{y}^{2} & u_{y y}^{2}
\end{array}\right|}{\left|\begin{array}{ll}
u_{x}^{1} & u_{y}^{1} \\
u_{x}^{2} & u_{y}^{2}
\end{array}\right|}, \quad I_{4}=\frac{\left|\begin{array}{ll}
u_{x}^{1} & u_{x y}^{1} \\
u_{x}^{2} & u_{x y}^{2}
\end{array}\right|-\frac{1}{2}\left|\begin{array}{ll}
u_{x x}^{1} & u_{y}^{1} \\
u_{x x}^{2} & u_{y}^{2}
\end{array}\right|}{\left|\begin{array}{ll}
u_{x}^{1} & u_{y}^{1} \\
u_{x}^{2} & u_{y}^{2}
\end{array}\right|}
\end{aligned}
$$

These four invariants are the generalizations of the standard Schwarz derivative, for the case of maps $u: \mathbb{R}^{2} \rightarrow \mathbb{R}^{2}$. The evolution (8.1) induces an 
evolution on the invariants $I_{1}, I_{2}, I_{3}, I_{4}$, which, as explained in the introduction, can be regarded as a generalization of the standard Hamiltonian $\mathrm{KdV}$ evolution - the Lie-Poisson evolution on the dual of the Virasoro algebra:

$$
I_{t}=h_{x x x}-2 I h_{x}-I_{x} h
$$

where $h$ is the Hamiltonian. In particular, the Korteweg-deVries equation is obtained when $h=I$.

But soon one realizes that we can never get a Hamiltonian evolution that way. For instance, (8.1) relies on a 2-dimensional vector of invariants $\left(J_{1}, J_{2}\right)$, whereas we now have 4 independent invariants. And even if we choose only two of them we can never find an independent Hamiltonian evolution: the invariants depend on the second jet of the surfaces, rather than on the third or higher jet, which happens in the case of curves. In view of these difficulties we need to find other (third order) invariants that will evolve according to a Hamiltonian system. For example, the three invariants:

$$
\begin{aligned}
I & =3\left(I_{1}\right)_{y}-2\left(I_{3}\right)_{x}-2 I_{3}^{2}-3 I_{1} I_{4}, \\
J & =3\left(I_{2}\right)_{y}-2\left(I_{4}\right)_{x}-2 I_{4}^{2}-3 I_{2} I_{3}, \\
K & =4\left(I_{4}\right)_{x}+4\left(I_{3}\right)_{y}+2 I_{3} I_{4}-\frac{9}{2} I_{1} I_{2} .
\end{aligned}
$$

will generate an entire family of Hamiltonian evolutions on the dual of the Virasoro algebra, one per direction on the $(x, y)$-plane. The following results can be found in [19].

Theorem 8.1. Given any combination $\partial_{z}=\alpha \partial_{x}+\beta \partial_{y}, \alpha, \beta \in \mathbb{R}$, there exists an invariant functional $E_{\alpha, \beta}=\alpha^{2} I+\alpha \beta K+\beta^{2} J$ such that, if $J_{1}=$ $\alpha h, J_{2}=\beta h$, and if $u$ evolves following equation (8.1), then $E_{\alpha, \beta}$ evolves following the KdV Hamiltonian evolution

$$
\left(E_{\alpha, \beta}\right)_{t}=h_{z z z}+2 E_{\alpha, \beta} h_{z}+\left(E_{\alpha, \beta}\right)_{z} h
$$

In this sense, the $E_{\alpha, \beta}$ can be viewed as generalizations of the traditional Schwarzian derivative.

As for classical Hamiltonian systems in two independent variables such as the KP equation, one finds one unavoidable complication: the nonlocal character of either the Hamiltonian functional or of the Poisson structure itself. 
Theorem 8.2. Consider I the invariant defined in (8.2). Then there exists an analytic function $h: \mathbb{R}^{2} \rightarrow R$ such that, if $J_{1}=I+h$ and $J_{2}=h$, and if $u$ evolves by (8.1), then I will satisfy the KP equation

$$
\left(I_{t}\right)_{x}=\left(I_{x x x}+3 I I_{x}\right)_{x}+\frac{3}{2} k^{2} I_{y y} .
$$

Of course, the function $h$ in the Theorem will never be a local functional on the jet space. We can't talk about the differential invariance of $h$, since it is not well defined for such purposes (a differential invariant is always a local functional on the jet space). That is, a more general theory would be necessary to include these nonlocal systems in the framework we have presented in this paper.

Further relationship of these evolutions with integrable partial differential equations and the underlying Poisson geometry is still under investigation.

\section{References.}

[1] M. Ackerman and R. Hermann, Sophus Lie's 1884 Differential Invariant Paper, Math Sci Press, Brookline, Mass., 1976.

[2] M. Adler, On a trace functional for formal pseudo-differential operators and the symplectic structure of the Korteweg-deVries type equations, Invent. Math. 50 (1979), 219-248.

[3] E. Cartan, La Méthode du Repère Mobile, la Théorie des Groupes Continus, et les Espaces Généralisés, Exposés de Géométrie No. 5, Hermann, Paris, 1935.

[4] E. Cartan, La Théorie des Groupes Finis et Continus et la Géométrie Différentielle Traitées par la Méthode du Repère Mobile, Cahiers Scientifiques, Vol. 18, Gauthier-Villars, Paris, 1937.

[5] E. Cartan, Sur la déformation projective des surfaces, in Oeuvres Complètes; part. III, vol. 1, Gauthier-Villars, Paris, 1955, 441-538.

[6] V.G. Drinfel'd and V.V. Sokolov, Lie algebras and equations of KdV type, J. Sov. Math. 30 (1985), 1975-2036.

[7] M. Fels and P.J. Olver, Moving coframes. I. A practical algorithm, Acta Appl. Math. 51 (1998), 161-213.

[8] M. Fels and P.J. Olver, Moving coframes. II. Regularization and theoretical foundations, Acta Appl. Math. 55 (1999), 127-208. 
[9] M. Fels and P.J. Olver, Moving frames and moving coframes, University of Minnesota, 1997.

[10] G. Fubini, Applicabilità proiettiva di due superficie, Rend. Circ. Mat. Palermo, 41 (1916), 135-162.

[11] I.M. Gel'fand and L.A. Dikii, A family of Hamiltonian structures connected with integrable nonlinear differential equations, in Collected Papers; I.M. Gel'fand, vol. 1, Springer-Verlag, New York, 1987.

[12] A. González-López, R. Hernández, and G. Marí Beffa, Invariant differential equations and the Adler-Gel'fand-Dikii bracket, J. Math. Phys. 38 (1997), 5720-5738.

[13] M.L. Green, The moving frame, differential invariants and rigidity theorems for curves in homogeneous spaces, Duke Math. J. 45 (1978), 735-779.

[14] P.A. Griffiths, On Cartan's method of Lie groups and moving frames as applied to uniqueness and existence questions in differential geometry, Duke Math. J. 41 (1974), 775-814.

[15] H.W. Guggenheimer, Differential Geometry, McGraw-Hill, New York, 1963.

[16] G.R. Jensen, Higher order contact of submanifolds of homogeneous spaces, Lecture Notes in Math., No. 610, Springer-Verlag, New York, 1977.

[17] B.A. Kupershmidt and G. Wilson, Modifying Lax equations and the second Hamiltonian structure, Inventiones Math. 62 (1981), 403-436.

[18] S. Lie, Über Differentialinvarianten, Math. Ann. 24 (1884), 537-578; also Gesammelte Abhandlungen, Vol. 6, B.G. Teubner, Leipzig, 1927, 95-138; see [1] for an English translation.

[19] G. Marí Beffa, The theory of differential invariance and infinite dimensional Hamiltonian evolutions, to appear in Banach Center Publ., Polish Ac. of Sc., Warsaw.

[20] G. Marí Beffa, The theory of differential invariants and KdV Hamiltonian evolutions, to appear in Bull. Soc. Math. France.

[21] P.J. Olver, Equivalence, Invariants, and Symmetry, Cambridge University Press, Cambridge, 1995.

[22] P.J. Olver, Moving frames and singularities of prolonged group actions, to appear in Selecta Math.

[23] A. Tresse, Sur les invariants différentiels des groupes continus de transformations, Acta Math. 18 (1894), 1-88. 
Differential invariants for parametrized projective surfaces

[24] E.J. Wilczynski, Projective differential geometry of curves and ruled surfaces, B.G. Teubner, Leipzig, 1906.

RECEIVEd November 20, 1997.

UNIVERSITY OF WISCONSIN

MADISON, WISCONSIN 53706

AND

UNIVERSITY OF MINNESOTA

MiNNEAPOLIS, MN 55455

E-mail addresses: maribeff@math.wisc.edu

olver@ima.umn.edu

http://math.umn.edu/ olver 